J. Clin. Chem. Clin. Biochem.

Vol. 17, 1979, pp. 605-607

\title{
Aktivitätsbestimmung des placentalen Isoenzyms der alkalischen Phosphatase im Serum: Ein Methodenvergleich
}

\author{
Von P. M. Bayer, F. Gabl und Eva Knoth
}

Aktivitätsbestimmung des placentalen Isoenzyms der alkalischen Phosphatase im Serum: Ein Methodenvergleich

(Eingegangen am 11. Dezember 1978/4. Mai 1979)

Zusammenfassung: Es wurden drei Methoden zur Bestimmung der Aktivität des placentalen Isoenzyms der alkalischen Phosphatase (EC 3.1.3.1) im menschlichen Serum verglichen:

1. Hitzeinaktivierung bei $65^{\circ} \mathrm{C}$ durch 10 Minuten,

2. Differentialinaktivierung mit $L$ - $p$-Bromtetramisol und

3. ein immunologischer Präzipitationstest.

Die Ergebnisse der drei Methoden korrelieren gut (Korrelationskoeffizienten zwischen 0,973 und 0,982). Für größere Serien oder zur Screeninguntersuchung auf das Vorkommen von „Regan-Isoenzym “ eignet sich die Methode der Differentialinaktivierung mit $L$ - $p$-Bromtetramisol wegen der kurzen Analysenzeit und der relativ einfachen Mechanisierbarkeit am besten.

\section{Measuring the activity of the placental isoenzyme of alkaline phosphatase: 3 methods compared}

Summary: Three methods were compared in human serum for determining the activity of the placental isoenzyme of alkaline phosphatase:

1. Heat inactivation at $65^{\circ} \mathrm{C}$ for 10 minutes,

2. Differential inactivation with $L$ - $p$-Bromotetramisole, and

3. an immunological precipation test.

A good comparison between the three methods was found (correlation coefficient between 0.973 and 0.982 ). For long series or screening determinations for "Regan-isoenzyme" the differential inactivation with $L-p$-Bromotetramisole is preferred because of the short analysis time and the possibility of simple mechanisation.

\section{Eintührung}

Dịe Aktivitätsbestimmung des placentạlen Isoenzyms der alkalischen Phosphatase (EC 3.1.3.1) gewinnt zunehmend an klinischem Interesse. Das placentale Isoenzym der alkalischen Phosphatase, das physiologischerweise in der Schwangerschaft im mütterlichen Serum auftritt, gilt als carcinoplacentales Antigen $(1,2)$. Es konnte bei einer Reihe von Patienten mit malignen Tumoren im Serum nachgewiesen werden (3). Mangels einfacher und mechanisierbarer Methoden sind Studien mit großen Probandenzahlen bisher nicht durchgefuihrt worden. Aus diesen Gründen ist es notwendig, wenig aufwendige Methoden zur quantitativen Bestimmung des genannten Isoenzyms der alkalischen Phosphatase zur Hand żu haben, die sich eventuell auch für ScreeningUntersuchungen eignen.
Im Folgenden stellen wir den Vergleich von 3 Methoden der Aktivitätsbestimmung des placentalen Isoenzyms der alkalischen Phosphatase dar, von denen eine den Anforderungen nach Einfachheit und Mechanisierbarkeit entspricht.

\section{Material und Methodik}

Zur Untersuchung kamen Sera von 31 schwangeren Frauen aus der zweiten Hälfte ihrer Gravidität.

Die Bestimmung der Aktivität der alkalischen Phosphatase erfolgte im optimierten Test (4) mit der Testkombination der

Fa. Boehringer (Mannheim) bei $25^{\circ} \mathrm{C}$ am Gemsaec (ElectroNucleonics). Die Aktivität des placentalen Isoenzyms wurde bestimmt durch:

\section{Hitżeinaktivierung (5)}

Die Sera wurden jeweils in $0,5 \mathrm{ml}$-Portionen in dünnwandigen Glasröhrchen (Durchmesser $0,5 \mathrm{~cm}$ ) durch 10 Minuten bei 
$65^{\circ} \mathrm{C}$ unter laufendem Schütteln inkubiert. Die Temperaturmessung erfolgte in einem der Röhrchen. Anschließend wurden die Proben rasch in Eiswasser abgekühlt und nach Temperaturausgleich mit der Umgebung $\left(20-22^{\circ} \mathrm{C}\right)$ analy siert. Die verbleibende Restaktivität entspricht dem placentalen Isoenzym der alkalischen Phosphatase.

\section{Differentialinaktivierung mit $L-p$-Bromtetramisol (6)}

$L$-p-Bromtetramisol (Aldrich, Europa) wurde in einer Endkonzentration von $50 \mu \mathrm{mol} / 1 \mathrm{dem}$ Testansatz zugefügt. Dieser $\mathrm{Zu}$ satz bewvirkt eine Hemmung des hepatalen und des ossären Isoenzyms der alkalischen Phosphatase um $90 \%$, während das placentale und das intestinale Isoenzym nur eine Hemmung von $10 \%$ auf weisen. Die Berechnung der Aktivität der placentalen alkalischen Phosphatase (bzw. des intestinalen Isoenzyms) erfolgte aus der Gesamtaktivität und der Aktivität nach der Hemmung nach Van Belle et al. (6).

\section{Immunologischer Präzipitationstest (7)}

Die zu untersuchenden Sera wurden mit Antiserum gegen placentale alkalische Phosphatase (für die Überlassung des Antiserums danken wir Herrn Dr. H. Lang, Fa. Merck, Darmstadt) entsprechend den Vorschriften des Herstellers inkubiert, das entstandene Präzipitat durch Zentrifugation aus dem Ansatz entfernt und die verbleibende Aktivität der alkalischen Phosphatase im Überstand gemessen. Aus der Differenz zwischen Gesamtaktivität und der Restaktivität im Ửberstand ergibt sich die Aktivität der Placentaphosphatase im Serum.

Da die eingesetzten Antikörper eine Kreuzreaktion mit intestinalem Isoenzym der alkalischen Phosphatase (7) zeigen, ist bei positivem Ausfall der Reaktion die Hitzeinaktivierung anzuschließen, was auch für Methode 2 (Hemmung mit $L$-p-Bromtetramisol) gilt.

\section{Ergebnisse}

1. Die Präzision der einzelnen Methoden war bei $\bar{x}=$ $50 \mathrm{U} / 1$ über 10 Tage: $\mathrm{VK}=9,5 \%$ (Hitzeinaktivierung.), $\mathrm{VK}=6,5 \%$ (immunologischer Präzipitationstest) bzw. VK $=4,8 \%$ ( $L$-p-Bromtetramisol).

2. Die Ergebnisse für den Methodenvergleich sind in Abbildung 1-3 graphisch dargestellt. Die Korrelation

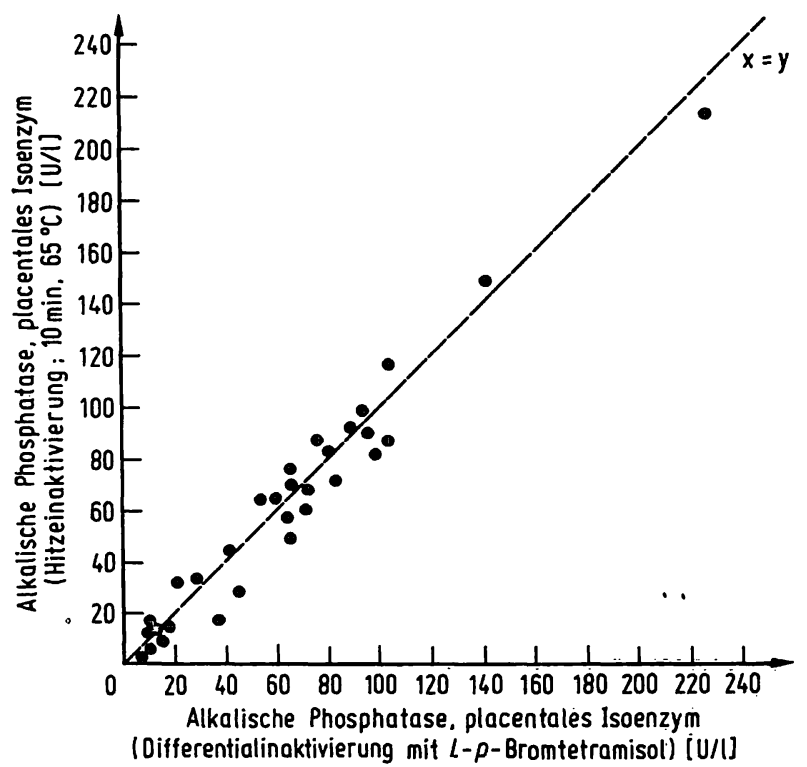

Abb. 1. Alkalische Phosphatase, placentales Isoenzym. Vergleich $z w i s c h e n$ Differentialinaktivierung mit $L$-p-Bromtetramisol und Hitzeinaktivierung. Methodik siehe Text. $\mathrm{N}=31$, $r=0,982, y=0,973, x-0,107$ zwischen Methode 1 (Hitzeinaktivierung) und Methode 2 (Hemmung mit $L$-p-Bromotetramisol) ist mit $r=0,982$ zufriedenstellend. Dies deckt sich mit den Angaben von Van Belle et al. (6). In Abbildung 2 (Methode 1 zu Methode 3), sowie in Abbildung 3 (Methode 2 zu Methode 3) sieht man, daß in den niedrigen Aktivitätsbereichen (unter $20 \mathrm{U} / \mathrm{l}$ ) der immunologische Präzipitationstest (Methode 3) jeweils höhere Ergebnisse lieferte als die Vergleichsmethoden. Die Korrelationskoeffizienten sind für den Vergleich der Methode $1 \mathrm{zu}$ Methode 3: $\mathrm{r}=0,982$ und für den Vergleich der Methode 2 zu Methode 3: $r=0,973$.

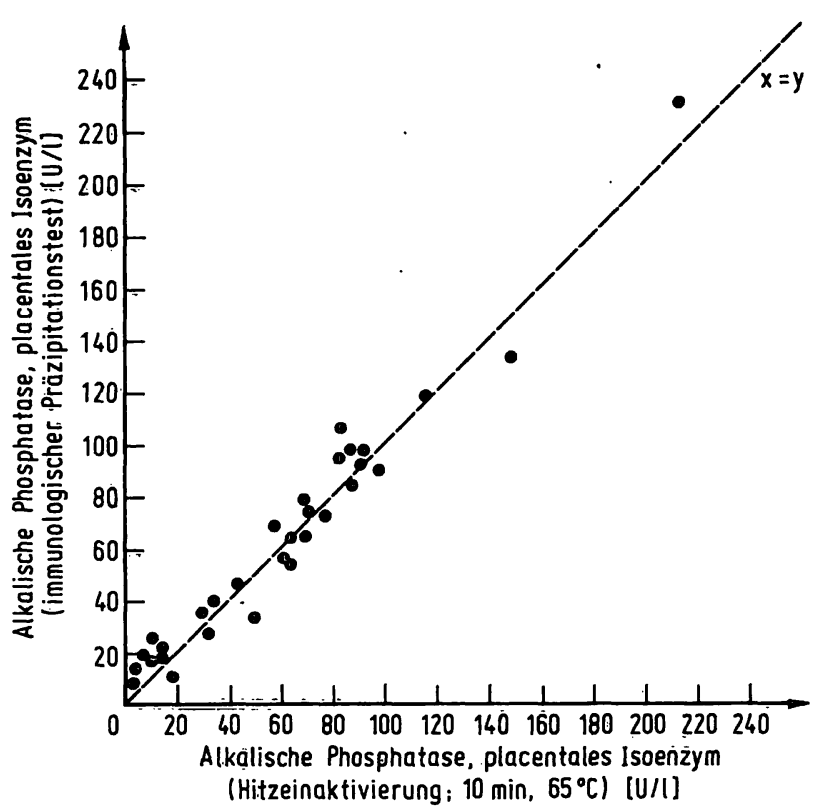

Abb. 2. Alkalische Phosphatase, placentales Isoenżym. Vergleịch zwischen Hitzeinak tivierung und immunologischem Präzipitationstest. Methodik siehe Text. N $=31, \mathrm{r}=0,982$, $y=0,988 x+3,823$

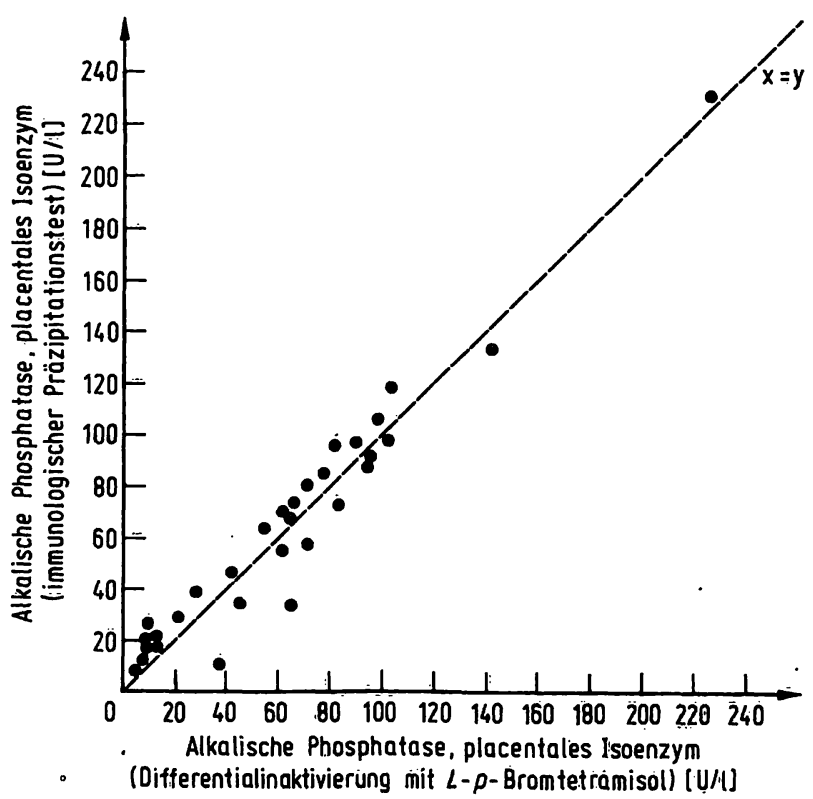

Abb. 3. Alkalische Phosphatase, placentales Isoenzym. Vergleich zwischen Differentialịnaktiviẹrung mit $L-p$-Bromtetramisol und immunologischem Präzipitationstest. Methodik siehe Text. $\mathrm{N}=31, \mathrm{r}=0,973, \mathrm{y}=0,970 \mathrm{x}+3,150$ 


\section{Diskussion}

Neben den elektrophoretischen Methoden der Isoenzymtrennung der alkalischen Phosphatase, die als Methoden 1. Ordnung nach Wieme (8) ihren festen Platz in der Isoenzymanalytik haben, sind andere Methoden zur quantitativen Bestimmung der einzelnen Isoenzymfraktionen notwendig. Insbesondere den Methoden, die einer Mechanisierung zugänglich sind, wird bei einer größeren Probenfrequenz Bedeutung zukommen.

Die Bestimmung der Aktivität des placentalen Isoenzyms der alkalischen Phosphatase mittels Hitzeinaktivierung gilt als Standardmethode. Die Ergebnisse hängen jedoch von mehreren, nur unter strengen Kautelen einhaltbaren Bedingungen ab: Exakte Temperaturkonstanz und Inkubationsdauer, völlig gleiche Wandstärke der zur Inkubation verwendeten Glasröhrchen, sowie Beachtung von pH-Schwankungen des Untersuchungsmaterials. Solche Schwankungen können bei gefroren gelagerten Proben auftreten. Ferner ist die Methode der Hitzeinaktivierung nur schwierig zu mecha-. nisieren.

Die Aktivitätshemmung des placentalen und des intestinalen Isoenzyms der alkalischen Phosphatase mittels $L$ Phenylalanin (9) weist ebenfalls einige Nachteile auf: In den üblichen Konzentrationen ist die Hemmung der genannten Isoenzyme nicht ausreichend (6), eine Parallel- inkubation mit $D$-Phenylalanin ist ferner notwendig. Für die Aktivitätsbestimmung des placentalen bzw. des intestinalen Isoenzyms der alkalischen Phosphatase mittels Phenylalaninhemmung sind somit 3 Ansätze und 3 Messungen notwendig. Die Hemmung der alkalischen Phosphatase-Aktivität durch EDTA soll alle Isoenzyme außer dem placentalen betreffen $(9,10)$. In unseren Händen hat sich diese Methode jedoch nur wenig bewährt.

Die hohe Spezifität der Bestimmung der placentalen alkalischen Phosphatase mittels spezifischer Antikörper wäre im Hinblick auf Untersuchungen der Placentafunktion während des Verlaufes einer Schwangerschaft $(11,12)$ und auf Screeninguntersuchungen nach dem "Regan-Isoenzym" der alkalischen Phosphatase (1, 2, 3) wünschenswert. Der uns zur Verfügung stehende Antikörper zeigte eine Kreuzreaktion mit dem intestinalen Isoenzym der alkalischen Phosphatase, so daß der Einsatz dieses Antiserums der Methode der Aktivitätshemmung mittels $L$-p-Bromtetramisol nicht überlegen ist: Sowohl die höheren Kosten, als auch der größere analytische und zeitliche Aufwand des immunologischen Präzipitationstests, bei gleicher Aussagekraft wie der in wenigen Minuten und mechanisiert durchführbare Differentialhemmtest mittels $L$ - $p$-Bromtestramisol, sprechen zur Zeit gegen den Einsatz der immunologischen Methode.

Die Untersuchungen erfolgten mit Unterstützung der EISNERStiftung.

\section{Literatur}

1. Fishmañ, W: H., Inglis, N. R., Green, S., Anstiss, C. L., Gosh, N. K., Reif, A. F., Rustigan, R., Krant, M. J. \& Stolbach, L. L. (1968), Nature 219, 697-699.

2. Fishman, W. H., Inglis, N. R. \& Green, S. (1971), Cancer Res. 31, 1054-1057.

3. Lehmann, F. G. (1975), Clin. Chim. Acta 65, 271-282.

4. Empfehlungen der Deutschen Gesellschaft für klinische Chemie (1972), J. Clin. Chem. Clin. Biochem. 10, 182-192

5. Moss, D. W. (1973), Chem. Rundschau 26, 11-16.

6. Van Bèlle, H., De Broe, M. E., Wieme, R. J. (1977), Clin. Chem. 23, 454-459.

7. Lehmannn, F. G. (1975), Clin. Chim. Actạ 65, 257-269.
8. Wieme, R. J. \& Demeulenaere, L. (1970), J. Clin. Pathol. 24, suppl. (Ass. Clin. Path.) 4, 51-59.

9. Wilkinson, J. H.: In „Isoenzymes“, Chapman and Hall Ltd. London 1970.

10. Conyers, R. A. J., Birkett, D. J., Neale, F. C., Posen, S. \& Brudenell-Woods, Joan. (1967), Biochim. Biophys. Acta 139, 363-371.

11. Rhone, D. P., Withe, Florence, M., Gidaspow, Helene (1974), Obstetr. Gynecol. 43, 31-40.

12. Marshall, B. R., Parisi, F. (1975), Obstetr. Gynecol. 45, 136-141.
Primarius Dr. P. M. Bayer Wilhelminenspital d. Stadt Wien Zentrallaboratorium

Mont leartstr. 37

A-1171 Wien

Österreich 